wird, seine Rechte als Vertragsarzt zu kennen. Aus Sicherstellungsgründen kann nach den Beschlüssen des Bewertungsausschusses nämlich auch weiterhin im Ein- zelfall von der Minderung des Fallwertes im Rahmen der Fallzahlbegrenzung abgewichen werden. Diesbezüglich sehen die Beschlüsse konkrete Anlässe vor, die auch weiterhin und natürlich nicht nur für die Fallwertstaffelung, sondern auch bei einer ggf. regional eingeführten zusätzlichen Regelung wie beschrieben gelten.

\title{
Begünstigter Steuersatz im Hotel: Was zählt dazu?
}

- Bei beruflich veranlassten Hotelaufenthalten gilt es, die Auswirkungen der gesetzlichen Neuregelungen, die zum 1.1.2010 in Kraft getreten sind, zu beachten. Dies betrifft insbesondere die Anwendung des ermäßigten Umsatzsteuersatzes für Beherbergungsleistungen $(\$ 12$ Abs. 2 Nr. 11 UStG) und die damit verbundenen lohnsteuerlichen Folgen in Bezug auf die Behandlung der Verpflegungsleistungen ab dem 1. Januar 2010.

Durch das Gesetz zur Beschleunigung des Wirtschaftswachstums vom 22. Dezember 2009 wurde $\S 12$ Abs. 2 UStG um eine neue Nummer 11 ergänzt. Danach unterliegen die Umsätze aus der Vermietung von Wohn- und Schlafräumen, die ein Unternehmer zur kurzfristigen Beherbergung von Fremden bereithält, dem ermäßigten Umsatzsteuersatz. Die Steuerermäßigung gilt allerdings nicht für Leistungen, die nicht unmittelbar der Vermietung dienen, auch wenn diese Leistungen mit dem Entgelt für die Vermietung abgegolten sind.

Sind in einer Rechnung neben der Beherbergungsleistung Sammelposten für andere, dem allgemeinen Umsatzsteuersatz unterliegende Leistungen einschließlich Frühstück ausgewiesen, und liegt keine Frühstücksgestellung durch den Arbeitgeber vor, so ist die "alte“ Vereinfachungsregelung (Abzug von 20\% des maßgebenden Pauschbetrags für Verpflegungsmehraufwendungen in Höhe von 4,80 Euro) auf diesen Sammelposten anzuwenden. Der verbleibende Teil dieses Sammelpostens kann als Reisenebenkosten im Sinne von R 9.8 der Lohnsteuerrichtlinien (LStR) 2008 durch den Arbeit-

\section{Info}

Nach einem Schreiben des BMF gehören folgende Leistungen auch zu den unmittelbaren Beherbergungsleistungen und unterliegen ebenfalls der Steuerermäßigung:

_ Überlassung von möblierten und mit anderen Einrichtungsgegenständen

(z. B. Fernsehgerät, Radio, Telefon, Zimmersafe) ausgestatteten Räumen

- Stromanschluss

_ Überlassung von Bettwäsche, Handtüchern und Bademänteln

- Reinigung der gemieteten Räume

Demgegenüber sind nachfolgende Leistungen keine Beherbergungsleistungen im Sinne von $§ 12$ Abs. 2 Nr. 11 UStG und daher auch nicht umsatzsteuerlich be-günstigt:

_ Überlassung von Räumen zur Ausübung einer beruflichen oder gewerblichen Tätigkeit

- Gesondert vereinbarte Überlassung von Plätzen zum Abstellen von Fahrzeugen

- Überlassung von Tagungsräumen

- Verpflegungsleistungen (z. B. Frühstück, Halb- oder Vollpension, „All inclusive”, Getränkeversorgung aus der Minibar)

- Nutzung von Kommunikationsnetzen (insbesondere Telefon und Internet)

- Nutzung von Fernsehprogrammen außerhalb des allgemeinen und ohne gesondertes Entgelt zugänglichen Programms (,pay per view“)

- Leistungen, die das körperliche, geistige und seelische Wohlbefinden steigern („Wellnessangebote“)

geber steuerfrei ersetzt werden. Da $a b$ dem 01.01.2010 in den Hotelrechnungen die Frühstückskosten separat ausgewiesen worden sind und in der Regel die Frühstückskosten den Pauschbetrag von 4,80 Euro erheblich übersteigen, können die Kosten des Frühstücks durch den Arbeitgeber übernommen und für den Arbeitnehmer nur der Sachbezugswert nach der Verordnung über die sozialversicherungsrechtliche Beurteilung von Zuwendungen des Arbeitgebers als Arbeitsentgelt (SVEV) für die Versteuerung in Höhe von 1,57 Euro angesetzt werden.

\section{MMW Kommentar}

Diese Verfahrensweise dürfte für den Arzt als Freiberufler selbst weniger relevant sein, aber z.B. bei von ihm bezahlten Fortbildungsveranstaltungen seines Per- sonals eine Rolle spielen. In diesem Fall liegt eine Arbeitgeberveranlassung vor, wenn die im Interesse des Arbeitgebers unternommene Auswärtstätigkeit zu der Übernachtung mit Frühstück führt und die Aufwendungen deswegen vom Arbeitgeber dienst- oder arbeitsrechtlich ersetzt werden. In diesem Fall muss die Rechnung auf den Arbeitgeber ausgestellt sein, der Arbeitgeber oder eine andere durch den Arbeitgeber dienst- oder arbeitsrechtlich beauftragte Person die Übernachtung mit Frühstück gebucht haben (z. B. über das elektronische Buchungssystem des Hotels) und eine entsprechende Buchungsbestätigung des Hotels vorliegen. Diesbezüglich sind allerdings Ausnahmen möglich, z.B. wenn eine dementsprechende planmäßige Buchung von Übernachtung mit Frühstück nicht möglich war. 